\title{
Synthesis and Antitumor Activity Evaluation of New 2-(4-aminophenyl) benzothiazole/oxazole/imidazole Derivatives
}

\author{
Elham Ezz El-Arab $^{1 \#}$, A. I. El-Said ${ }^{1}$, M. S. Amine ${ }^{2}$ and H. \\ H. Moharram ${ }^{1}$ \\ ${ }^{1}$ Department of Chemistry, NODCAR (National Organization \\ for Drug Control and Research), Giza 12311 and \\ ${ }^{2}$ Chemistry Department, Faculty of Science, Banha University, \\ Benha, Egypt.
}

\begin{abstract}
A NEW series of 2-(4-aminophenyl)benzothiazole and related A structure compounds, bearing thiocyanate, thiol in the 3 postion as well as new condensed benzothiazoles and related structure compounds having thiazolones ,tetrazole, oxadiazole have been synthesized. All the newly synthesized compounds were screened for their anti-tumor activities. The results revealed promising effects against most of the cancer cell lines.
\end{abstract}

Keywords: 2-(4 aminophenyl) benzothiazole/ benzoxazole/ benzimidazole, Tetrazole, oxadiazole, Thiazole, Oxobutanoate, Tolyiformamidine, Chlorophenylformamidine, Anticancer agent.

The relationship between chemical and biological activity was found of interest to pharmacologists and medicinal chemists, it was the basic of attempts to design compounds of therapeutic value. The structural simplicity and synthetic accessibility of benzothiazole ${ }^{(1-4)}$, benzoxazole and benzimidazole series show remarkable antitumor properties. A series of potent and selective agents derived from 2-(4-Aminophenyl)benzothiazole structure was extensively examined and developed during recent years to have antitumor activity since $1996^{(5-19)}$, Unexpectedly, it was found that , 2-(4-aminophenyl) benzothiazole and related structure derivatives inhibit cancer cell growth with nanomolar scale against a large panel of human cancer cell lines particularly against breast, colon and ovarian cell lines in in-vitro anticancer screening program of the National Cancer Institute (NCI) with a characteristic biphasic dose-response relationship. The original (unsubstituted) member of this series, 2-(4-aminophenyl) benzothiazole was considered to be "Lead "approach to drug discovery of new anticancer agents. They are, therefore, used as important intermediates for synthesis of a variety of potent commercial drugs ${ }^{(20-22)}$.For example, chemical structures of these drugs here pimobendan (ionodilator), mebendazole (anthemintic) , phortress, Albendazole and

\footnotetext{
${ }^{\#}$ Corresponding author e-mail: elhamezz@ hotmail.com.
} 
Omeprazole. Phortress8 (NSC 710305) as shown in Fig.1. Motivated by the above observations and as extension of on our previous study ${ }^{(23-24)}$, we planned to synthesize new series of 2-(4-aminophenyl) benzothiazole derivatives including ( benzimidazole / benzoxazole/ benzothiazole substituted in the 3position of the phenyl ring by thiocyanate or thiol substituents. All the newly synthesized compounds were evaluated for their antitumor activity against nine cancer types comprised of approximately 60 cell lines at NCI at USA.<smiles>CC(C)(C)[C@@](C)(CN)C(=O)Nc1ccc(-c2nc3cc(F)ccc3s2)cc1</smiles><smiles>CCCSc1ccc2nc(NC(=O)OC)[nH]c2c1</smiles>

albendazole<smiles>COC(=O)Nc1nc2ccc(C(=O)c3ccccc3)cc2[nH]1</smiles>

mebendazole<smiles>COc1ccc2nc(S(=O)Cc3ncc(C)c(OC)c3C)[nH]c2c1</smiles><smiles>COc1ccc(-c2nc3cc(C4=NNC(=O)CC4C)ccc3[nH]2)cc1</smiles>

Fig.1.

Chemistry

\section{Results and Discussion}

The key starting material 2-(4-aminophenyl) benzothiazole and its structures $(1 \mathrm{a}-\mathrm{c}) \quad$ were prepared as previously reported ${ }^{(25)}$ by the condensation of 4-aminobenzoic acid with 2-aminothiophenol, 2-aminophenol or 1, 2phenylenediamine, respectively, in presence of polyphosphoric acid at high temperature.

One-pot reaction of appropriate 1a-c with thiocyanogen generated from bromine and alkaline thiocyanate in acetic acid medium afforded different compounds. So, when the reaction was carried out at zero temps for $3 \mathrm{hr}$. The compounds $2 \mathrm{a}-\mathrm{c}$ thiocyanate benzeneamine were formed.

On the other hand, new amino thiazole derivatives 4a-c were formed when the reaction was carried out with stirring at room temperature for $24 \mathrm{hr}$. Also, it were formed when compounds $2 \mathrm{a}-\mathrm{c}$ was heated in acetic acid at $60^{\circ} \mathrm{C}$ for $3 \mathrm{hr}$ to give $4 \mathrm{a}-\mathrm{c}$. When compound $2 \mathrm{a}-\mathrm{c}$ was reacted with sodium sulfide in ethyl alcohol, substitution of the thiocyanate by thiol was occurred at 3postion of 2-phenyl group to afforded 3a-c derivatives. The latter compounds 
were reacted with ethyl cyanoacetate at $140^{\circ} \mathrm{C}$ for 3 hours to give thiazol-2acetonitrile derivatives $5 \mathrm{a}, \mathrm{b}$ as shown in Scheme1.

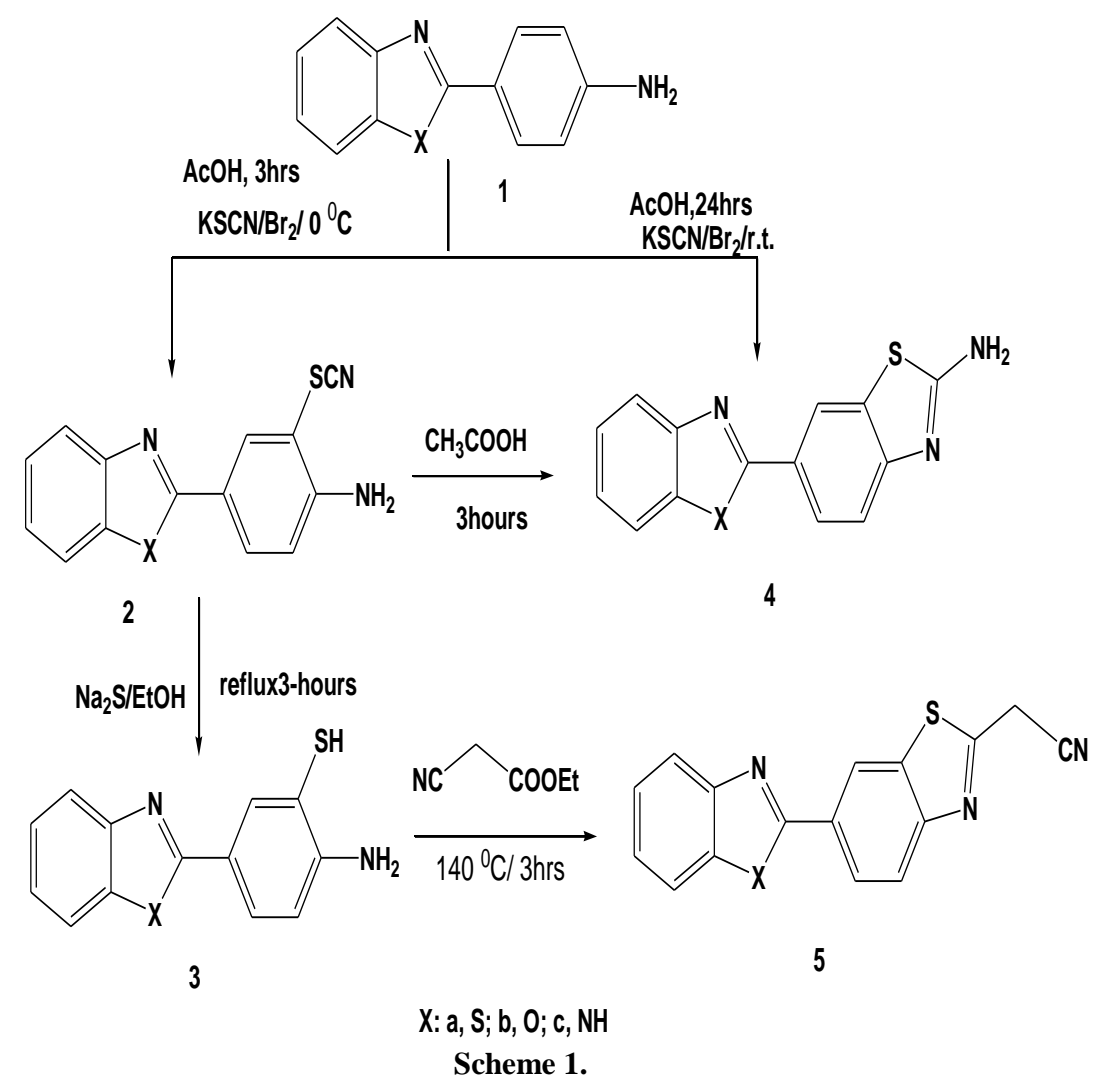

Moreover, the action of copper (I) cyanide on diazonium salts of compounds $1 \mathrm{a}-\mathrm{c}$ at $60-70^{\circ} \mathrm{C}$ for $15 \mathrm{~min}$ furnished compounds $6 \mathrm{a}, \mathrm{b}$ through the Sandmeyer reaction. Cyclocondensation occurred between the latter compounds with thioglycolic acid in boiling glacial acetic acid to form thiazolidin-4-one derivatives $7 \mathrm{a}-\mathrm{c}$ as shown in (Scheme2).

On the other hand, reaction of $6 \mathrm{a}, \mathrm{b}$ with sodium azide and ammonium chloride in DMF gave tetrazoles analogs of benzothiazole/ benzoxazole structures of compounds 8a, b. 2-Methyl -1, 3, 4- oxadiazoles derivatives $9 \mathrm{a}, \mathrm{b}$ were prepared through thermal rearrangement of compound 8 by heating with acetic anhydride - The reaction was represented as ring openings of tetrazoles to give intermediates which loss nitrogen to give new oxdiazoles rings ${ }^{(26)}$. When 2-(4-aminophenyl) benzothiazole and its derivatives $1 \mathrm{~b}, \mathrm{c}$ 
reacted with 3- methylpyrazolone and triethyl orthoformate gave methylene $-3-1 \mathrm{H}$ - pyrazolo- 5 - $(4 \mathrm{H})$ - one compounds $10 \mathrm{~b}, \mathrm{c}$. Also, $1 \mathrm{a}, \mathrm{b}$ reacted with triethyl orthoformate and active methylene aliphatic compounds as malononitrile, ethyl cyanoacetate, ethyl acetoacetate and afforded methylenecyanoacrylate (oxobutanoate) derivatives 11a-d. As well as $1 \mathrm{a}, \mathrm{b}$ reacted with aromatic amino compounds and triethylorthoformate and gave formamidine derivatives 12a-e as shown in Scheme 3. The structures of the synthesized compounds were assigned on the basis of elemental analysis ,IR, ${ }^{1} \mathrm{H}-\mathrm{NMR}$ and mass spectra data .The compounds were screened for their in-vitro antitumor activity.

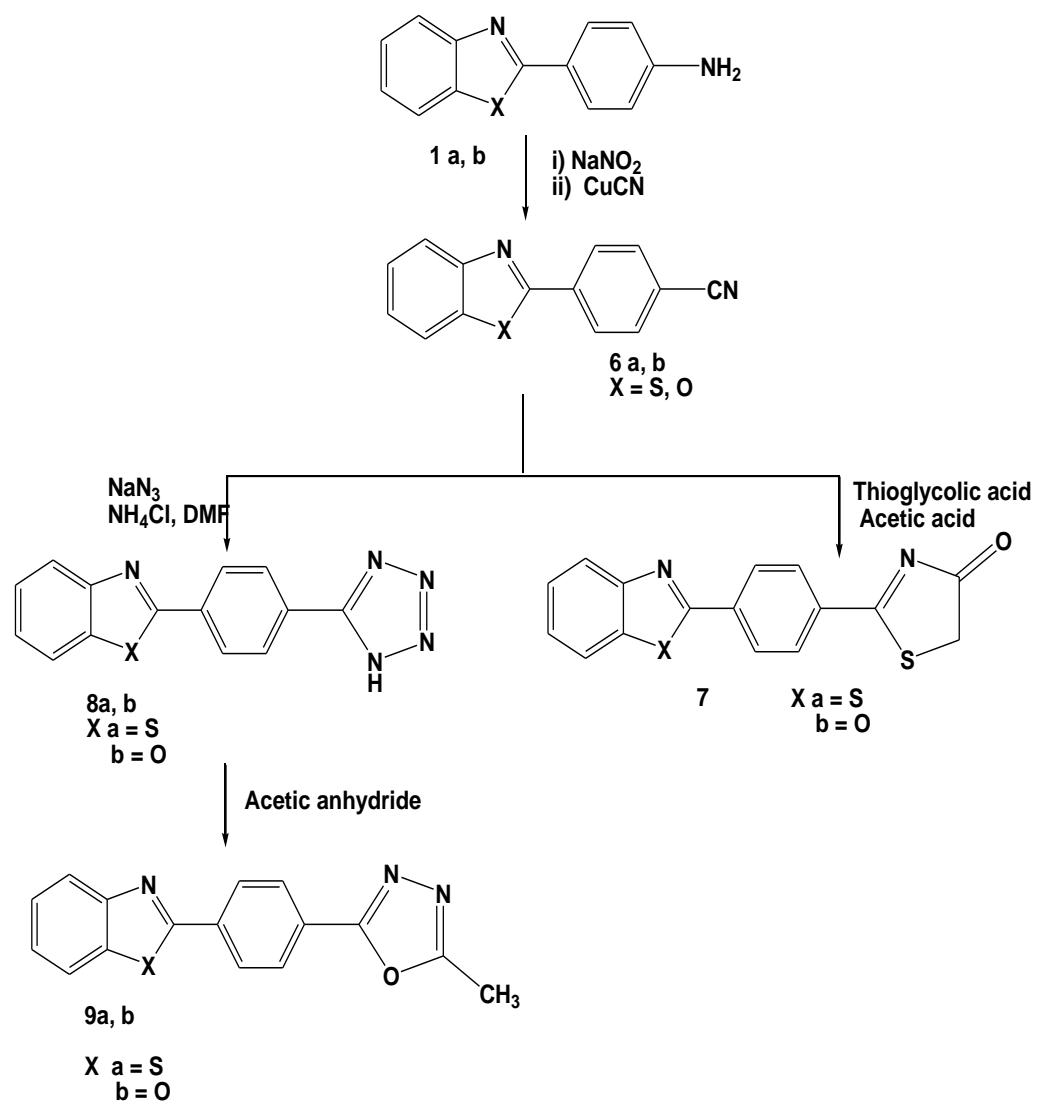

Scheme 2

Egypt. J. Chem. 59, No. 6 (2016) 


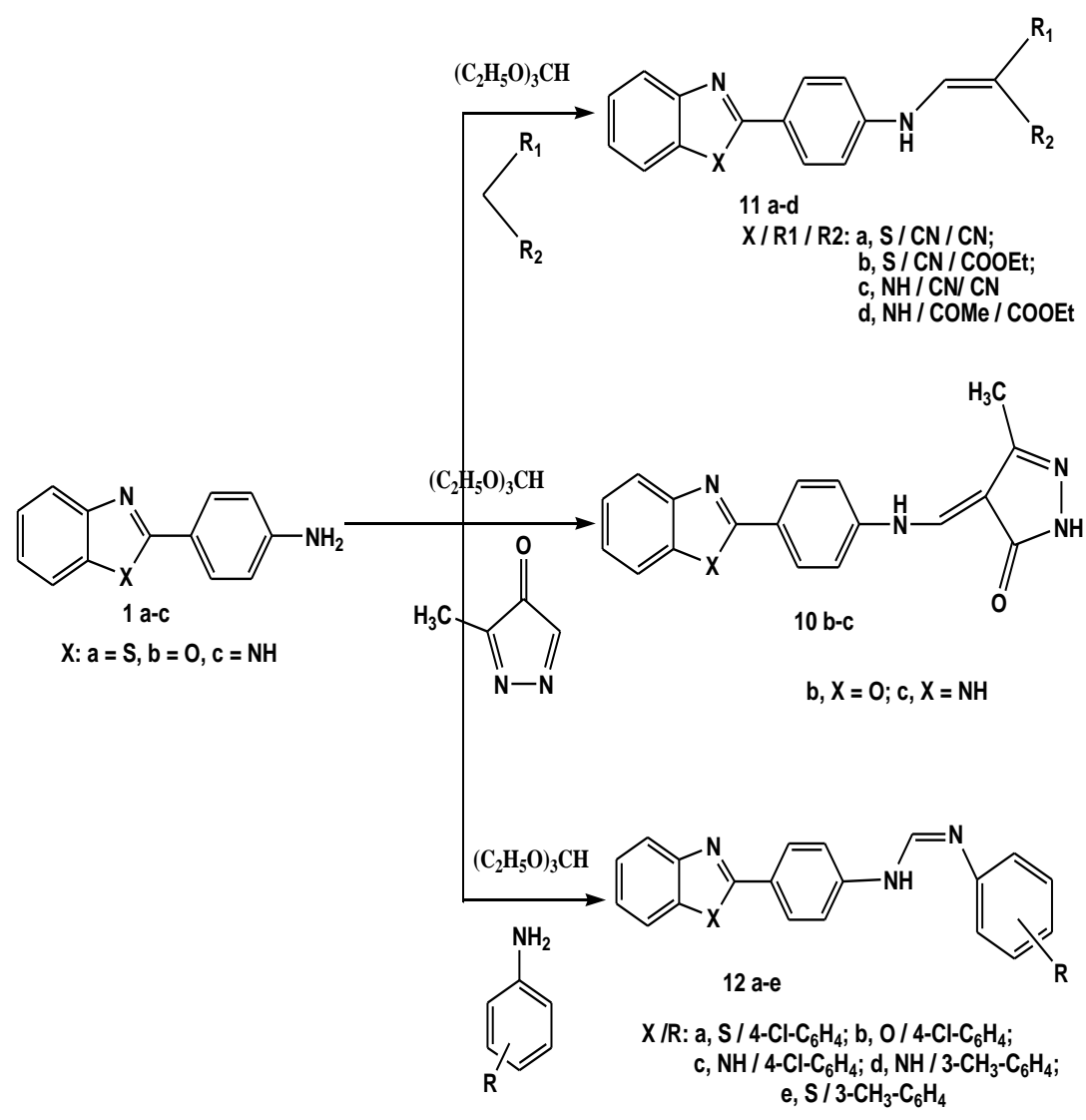

Scheme 3.

Biological screening (antitumor activity)

The hetero cyclic compounds, prepared in this study, were evaluated according to their cytotoxic and /or growth inhibitory effects of the compounds were evaluate in vitro against approximately 60 human tumor cell lines derived from nine neoplastic diseases ,namely: leukemia(L), non-small cell lung cancer(NSCLC), colon cancer (CC), central nervous system cancer (CNSC), melanoma(M), ovarian cancer(OC), renal cancer (RC),prostate cancer(PC) and breast cancer (BC) at NCI, Bethesdas, USA. The in vitro screening program was based upon the use of multiple panels of 60 human tumor cell lines, against which the compounds were tested at tenfold dilutions of five concentrations ranging from $10^{-4}$ to $10^{-8} \mathrm{M}$. After incubation of 48-h continuous drug exposure protocol was followed, and a sulforhodamine B protein assay was used to estimate cell growth ${ }^{(27-29)}$. All the newly synthesized compounds were offered for testing their anticancer activity according to in-vitro drugs screening

Egypt. J. Chem. 59, No.6 (2016) 
protocol of the institute. Compounds $2 b, 3 b, 7 a, c, 9 b, 10 c, 12 a, b$ and $12 c$ were carried out against 60 human tumor cell lines drived from nine cancer types L,NSCLC, CC,CNS, RC, PC and BC.

The results were reported both as dose response curves and pictographically as "mean graphs" compounds were consider to be active only if their $\operatorname{LogGI}_{50}$ values less than -4.5 compared to 5-Fluorouracil(5-Flu) the NCI standard anticancer agent.

The obtained data revealed that most of the newly synthesized compounds showed potent antitumor activity as shown in Table 1 .

Both compounds possess $\log \mathrm{GI}_{50}$ values lawer than -4.5 or-5,showing a notable activity level. Among the tested compounds, compound 10c demonstrate higher activity than standard against the following cell lines L and $\mathrm{BC} \operatorname{LogGI}_{50}-7.18$ and -8.0 respectively as shown in Fig.2.

Also, Compound $2 \mathrm{~b}$ showed higher activity than standard against the following cell line CNS and L. $\log \mathrm{GI}_{50}-7.16$ and -6.6 , respectively as shown in Fig.3.

TABLE 1. In-vitro disease-oriented anti-tumor screening.

\begin{tabular}{|l|c|c|c|c|c|c|c|c|c|}
\hline $\begin{array}{l}\text { *No. } \\
\text { Comp. }\end{array}$ & $\mathbf{L}$ & $\mathbf{C N S}$ & NSCL & $\mathbf{B C}$ & $\mathbf{M C}$ & $\mathbf{C C}$ & $\mathbf{R C}$ & OC & PC \\
\hline $\mathbf{2 b}$ & -6.66 & -7.16 & -5.42 & -4.86 & $\mathrm{NT}$ & -4.87 & -4.91 & $\mathrm{NT}$ & -5.06 \\
\hline $\mathbf{3 b}$ & -5.11 & -4.97 & -4.93 & -5.21 & $\mathrm{NT}$ & -5.09 & -4.94 & -5.12 & -5.16 \\
\hline $\mathbf{7 a}$ & -4.88 & -4.55 & -5.74 & -5.12 & -5.13 & -5.51 & $\mathrm{NT}$ & -4.80 & $\mathrm{NT}$ \\
\hline $\mathbf{7 c}$ & -5.24 & -4.53 & -5.49 & 5.05 & -4.80 & -4.78 & -4.76 & -4.64 & -4.50 \\
\hline $\mathbf{9 b}$ & -4.59 & -4.79 & -4.58 & $\mathrm{NT}$ & -4.81 & $\mathrm{NT}$ & $\mathrm{NT}$ & $\mathrm{NT}$ & $\mathrm{NT}$ \\
\hline $\mathbf{1 0 c}$ & -7.18 & -4.84 & -4.70 & -8.0 & $-\mathrm{NT}$ & -4.74 & $\mathrm{NT}$ & -4.83 & $\mathrm{NT}$ \\
\hline $\mathbf{1 2 a}$ & -5.02 & -5.11 & -5.40 & -5.03 & -6.84 & -5.02 & -4.93 & -5.03 & -4.78 \\
\hline $\mathbf{1 2 b}$ & -4.63 & -4.98 & -5.40 & -5.03 & -5.02 & -4.96 & -4.93 & -4.95 & -5.72 \\
\hline $\mathbf{1 2 c}$ & -6.20 & -4.68 & -6.32 & -5.58 & -6.41 & -6.16 & -6.08 & -5.27 & $\mathrm{NT}$ \\
\hline $\mathbf{5 - F U ^ { a }}$ & -6.25 & -5.87 & -5.70 & -5.47 & -6.03 & -6.73 & -6.24 & -5.74 & -6.03 \\
\hline
\end{tabular}

$\operatorname{LogGI}_{50}:$ Log concentration which able reduce cell growth to 50\% (a).NCI's data for 5-Flourouracil NSC 19893( NCI standard compound.). 


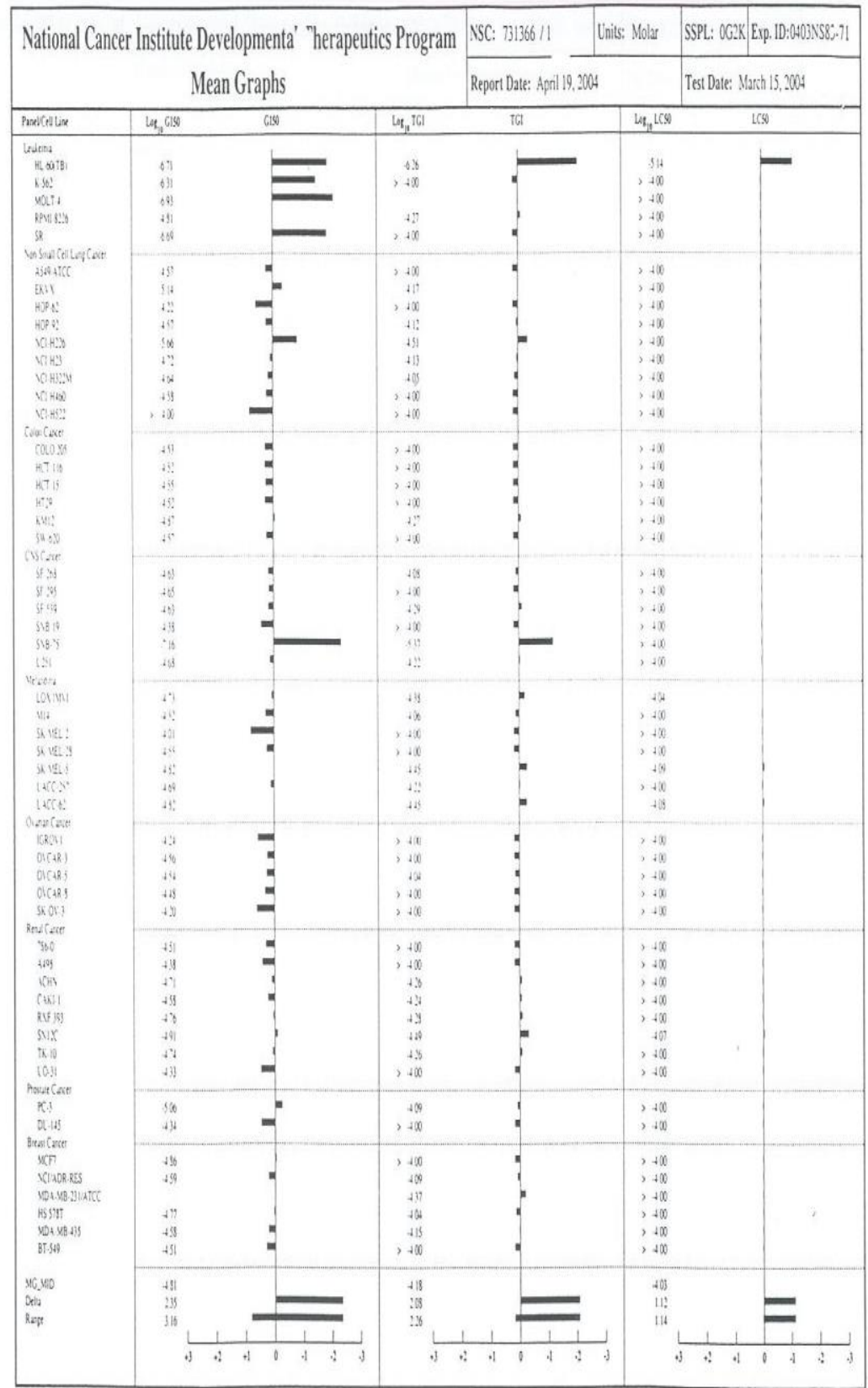

Fig. 2. It represent higher activity for compound $10 \mathrm{c}$ than standard against the following cell lines $\mathrm{L}$ and BC LogGI50 $\mathbf{- 7 . 1 8}$ and $\mathbf{- 8 . 0}$, respectively

Egypt. J. Chem. 59, No.6 (2016) 


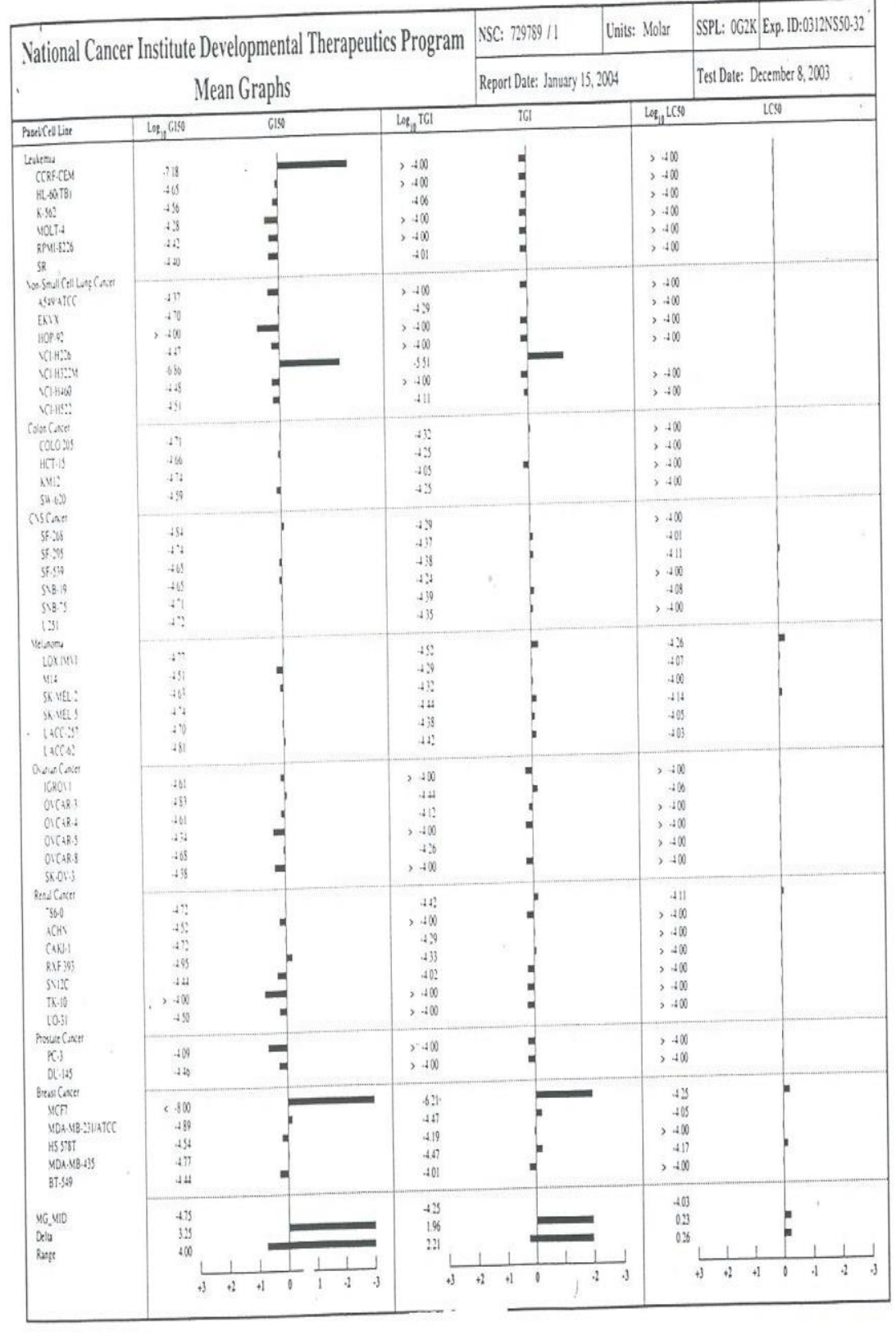

$\ln c$

Fig.3. Compound 2b showed higher activity than standard against the following cell line CNS and L. LogGI50-7.16 and -6.6, respectively.

Egypt. J. Chem. 59, No. 6 (2016) 


\section{Experiment}

\section{Experimental procedure}

The structure of all the synthesized compounds are confirmed by various spectral studies. All melting points are uncorrected and determined by the open capillary method using Gallen Kamp melting point apparatus. Microanalysis were carried out by the Micro Analytical unit at Cairo University. IR spectra $\mathrm{KBr}$ disk were recorded on FTIR-300E Jasco spectro- photometer. ${ }^{1} \mathrm{HNMR}$ spectra were recorded on a Varian EM $200 \mathrm{MHz}$ using (TMS as internal reference). Mass spectra were taken on Cairo University GC MS-QP/1000 EX(Shimadzu). All the results were in an acceptable range. Compounds 1a-c were synthesized according to the reported method $^{(25)}$.

General method for the synthesis of compounds (2a-c)

A mixture of appropriate 2-(4-aminophenyl) benzothiazol, benzoxazol, benzimidazole 1a-c $(0.01 \mathrm{~mol})$, potassium thiocyanate $(0.01 \mathrm{~mol})$ and bromine $(0.01 \mathrm{~mol})$ in glacial acetic acid $(40 \mathrm{ml})$ was stirred for $3 \mathrm{hr}$ at $0^{\circ} \mathrm{C}$ then the reaction was allowed to cool to room temp. and poured into water. The precipitate was collected and washed several times with water and recrystallized from ethanol to give $2 \mathrm{a}-\mathrm{c}$.

4-(benzo[d]thiazol-2-yl)-2-thiocyanatobenzenamine (2a)

Yellow solid, (80\% yield), m.p. $88-90{ }^{\circ} \mathrm{C}$; IR ( $\left.\mathrm{KBr}\right) v_{\max } 3449,3410$ $\left(\mathrm{NH}_{2}\right), 3053$ (aromaticC-H), $2212(\mathrm{CN}), 1600,1575,1445$ (CH-aromatic) $\mathrm{cm}^{-1}$; ${ }^{1} \mathrm{H}$ NMR (DMSO- $\left.d_{6}\right) \delta: 3.56\left(\mathrm{~s}, 2 \mathrm{H}, \mathrm{NH}_{2}\right), 6.36(\mathrm{~s}, 1 \mathrm{H}$, aromatic-H6), 7.3-8.12 (m, 6H, ArH's); Anal. Calcd. for $\mathrm{C}_{14} \mathrm{H}_{9} \mathrm{~N}_{3} \mathrm{~S}_{2}$ (283.37): C, 59.34; H, 3.20. Found: C, $59.04 ; \mathrm{H}, 3.53 \%$.

\section{4-(benzo[d]oxazol-2-yl)-2-thiocyanatobenzenamine (2b)}

White solid, (65\% yield), mp $129-130{ }^{\circ} \mathrm{C}$; IR ( $\left.\mathrm{KBr}\right) v_{\max } 3443,3400\left(\mathrm{NH}_{2}\right)$, $3067\left(\right.$ aromatic-CH) $, 2155(\mathrm{CN}), 1600,1575,1445(\mathrm{CH}$-aromatic $) \mathrm{cm}^{-1} ;{ }^{1} \mathrm{H}$ NMR $\quad\left(\right.$ DMSO- $\left.d_{6}\right) \quad \delta: 5.6\left(\mathrm{~s}, 2 \mathrm{H}, \mathrm{NH}_{2}\right), 6.36(\mathrm{~s}, 1 \mathrm{H}$, aromatic-H5),7.01-715(m,2H,ArH),7.26-7.20(m,4H,ArH); MS; m/z (\%) $267\left(\mathrm{M}^{+}, 100\right), 269\left(\mathrm{M}^{+2}\right.$, 7.7) $,(209,1.3),(119,1.6),(93,2.0)$ Anal. Calcd. for $\mathrm{C}_{14} \mathrm{H}_{9} \mathrm{~N}_{3} \mathrm{OS}(267.31) \mathrm{C}$, $62.91 ; \mathrm{H}, 3.39$; Found: C, 59.04; H, 3.53\%.

4-(1H-benzo[d]imidazol-2-yl)-2-thiocyanatobenzenamine (2c)

Yellow solid, (68\% yield), mp 213-215 ${ }^{\circ}$; IR ( $\left.\mathrm{KBr}\right) v_{\max } 3422,3410\left(\mathrm{NH}_{2}\right)$, $3321(\mathrm{NH}), 3174$ (aromatic- $\mathrm{CH}), 2218(\mathrm{CN}), 1600,1575,1445$ (CH-aromatic) $\mathrm{cm}^{-1} ;{ }^{1} \mathrm{H}$ NMR (DMSO- $\left.d_{6}\right) \delta: 4.3\left(\mathrm{~s}, 2 \mathrm{H}, \mathrm{NH}_{2}\right), 6.5-7.2(\mathrm{~m}, 3 \mathrm{H}, \mathrm{ArH}), 7.25-7.50$ $\left(\mathrm{m}, 4 \mathrm{H}\right.$, benzimidazol-H), $10.73(\mathrm{~s}, 1 \mathrm{H}, \mathrm{NH})$; Anal. Calcd. for $\mathrm{C}_{14} \mathrm{H}_{10} \mathrm{~N}_{4} \mathrm{~S}$ (266.32): C, 63.14; H, 3.78; Found: C, 63.44; H, 3.53\%.

General method for synthesis of compounds (3a-c).

A solution of 2a-c $(0.01 \mathrm{~mol})$ in ethyl alcohol $(50 \mathrm{ml})$ and sodium sulphide $(0.01 \mathrm{~mol})$ in $(20 \mathrm{ml})$ water was heated on water bath for one hour, then allowed 
to cool at room temperature, and poured into water, acidified with acetic acid. The precipitate was collected and washed several times with water then with ether and re-crystallized from ethyl alcohol to give 3a-c.

2-amino-5-(benzo [d] thiazol-2-yl) benzenethiol (3a)

Yellow solid, (50\% yield), mp 283-285 ${ }^{\circ} \mathrm{C}$; IR ( $\left.\mathrm{KBr}\right) v_{\max } 3366\left(\mathrm{NH}_{2}\right)$, 3053 (aromatic-CH) ,2368 (SH), 1600, 1575, $1445\left(\mathrm{CH}\right.$-aromatic) $\mathrm{cm}^{-1} ;{ }^{1} \mathrm{H}$ NMR (DMSO- $\left.d_{6}\right) \delta: 2.5(\mathrm{~s}, 1 \mathrm{H}, \mathrm{SH}), 5.20\left(\mathrm{~s}, 2 \mathrm{H}, \mathrm{NH}_{2}\right), 6.50(\mathrm{~s}, 1 \mathrm{H}$, aromaticH5), 7.40-7.48 (m, 6H, Ar-H); Anal. Calcd. for $\mathrm{C}_{13} \mathrm{H}_{10} \mathrm{~N}_{2} \mathrm{~S}_{2}$ (258.03): C, 60.43; H, 3.90. Found: C, 60.75; H, 3.55.\%.

2-amino-5-(benzo[d]oxazol-2-yl) benzenethiol( $3 b$ )

Yellow solid, (55\% yield), $\mathrm{mp} 283-285{ }^{\circ} \mathrm{C}$; IR (KBr) $v_{\max } 3326$ $\left(\mathrm{NH}_{2}\right), 3174$ (aromatic-CH), $2212(\mathrm{SH}) \quad \mathrm{cm}^{-1} ;{ }^{1} \mathrm{H}$ NMR (DMSO- $\left.d_{6}\right) \quad \delta$ : 3.0 $(\mathrm{s}, 1 \mathrm{H}, \mathrm{SH}), 6.41\left(\mathrm{~d}, 2 \mathrm{H}, \mathrm{NH}_{2}\right), 6.44(\mathrm{~s}, 1 \mathrm{H}$, aromatic-H5), 6.95-7.95 $(\mathrm{m}, 6 \mathrm{H}$, Ar-H); Anal. Calcd. for $\mathrm{C}_{13} \mathrm{H}_{10} \mathrm{~N}_{2} \mathrm{OS}$ (242.05): C, 64.44; H, 4.16 Found: $\mathrm{C}$, $64.75 ; \mathrm{H}, 4.74 \%$.

2-amino-5-(1H-benzo[d] imidazol-2-yl)benzenethiol(3c)

White solid, (50\% yield), mp $172-175{ }^{\circ} \mathrm{C}$; IR ( $\left.\mathrm{KBr}\right) \quad v_{\max } 3316$ $\left(\mathrm{NH}_{2}\right), 3049$ (aromatic-CH), $2216(\mathrm{SH}), 1600,1575,1445$ (CH-aromatic) $\mathrm{cm}$ ${ }^{1}$; ${ }^{1} \mathrm{H}$ NMR (DMSO- $\left.d_{6}\right) \delta 2.8(\mathrm{~s}, 1 \mathrm{H}, \mathrm{SH}), 5.91\left(\mathrm{~s}, 2 \mathrm{H}, \mathrm{NH}_{2}\right), 6.68(\mathrm{~s}, 1 \mathrm{H}$, aromatic-H5), 7.40-7.48 (m, 6H, Ar-H),10.73(s,1H,NH); Anal. Calcd. for $\mathrm{C}_{13} \mathrm{H}_{11} \mathrm{~N}_{3} \mathrm{~S}$ (241.07): C, 64.70; H, 4.59; Found: C, 65.02; H, 4.29\%.

General method for synthesis of $(4 a, b)$

A mixture of $1 \mathrm{a}, \mathrm{b}(0.01 \mathrm{~mol})$, potassium thiocyanate $(0.01 \mathrm{~mol})$ and bromine (0.01) in glacial acetic acid $(40 \mathrm{ml})$ was stirred at room temperature for $21 \mathrm{hr}$, then poured into water, the precipitate was collected and washed several times with water then with ether and recrystallized from ethanol to give $4 a, b$.

6-(benzo[d]thiazol-2-yl)benzo[d]thiazol-2-amine(4a)

Yellow solid, (65\% yield), m.p. $199-200{ }^{\circ} \mathrm{C}$; IR (KBr) $v_{\max } 3366$ $\left(\mathrm{NH}_{2}\right), 3067$ (aromatic-CH) $1600,1575,1445\left(\mathrm{CH}\right.$-aromatic) $\mathrm{cm}^{-1} ;{ }^{1} \mathrm{HNMR}$ $\left(\right.$ DMSO- $\left.d_{6}\right) \delta: 5.91\left(\mathrm{~s}, 2 \mathrm{H}, \mathrm{NH}_{2}\right), 7.48(\mathrm{~m}, 6 \mathrm{H}, \mathrm{Ar}-\mathrm{H}), 8.29(\mathrm{~d}, 1 \mathrm{H}$, aromatic-H5); MS: $\mathrm{m} / \mathrm{z}^{\prime} \mathrm{s} 283\left(\mathrm{M}^{+}, 100 \%\right), 284\left(\mathrm{M}^{+1}, 18.6 \%\right), 285\left(\mathrm{M}^{+2}, 10.0 \%\right),(267,1.9 \%)$; Anal. Calcd. for $\mathrm{C}_{14} \mathrm{H}_{9} \mathrm{~N}_{3} \mathrm{~S}_{2}$ (283.37.37): C, 59.34; H, 3.20. Found: C, 60.75; $\mathrm{H}, 3.55 \%$.

6-(benzo[d]oxazol-2-yl)benzo[d]thiazol-2-amine(4b)

Yellow solid, (60\% yield), m.p. $209-210^{\circ} \mathrm{C}$; IR $(\mathrm{KBr}) \mathrm{cm}^{-1} ; 3366\left(\mathrm{NH}_{2}\right)$, 3067(aromatic-CH), 1575(aromatic ring); ${ }^{1} \mathrm{H}$ NMR(DMSO-d6) $\delta: 6.4$ (s, 2H, $\left.\mathrm{NH}_{2}\right), 7.13-8.30(\mathrm{~m}, 7 \mathrm{H}, \mathrm{ArH}), 8.30\left(\mathrm{~d}, 1 \mathrm{H}\right.$, aromatic-H-5). Ms:m/ z's $\left(\mathrm{M}^{+}\right.$, $100 \%), 268\left(\mathrm{M}^{+1}, 14.1 \%\right)$, $(192,15.8 \%),(118,4.5 \%),(92,11.3)$; Anal . 
Calculated for $\mathrm{C}_{14} \mathrm{H}_{9} \mathrm{~N}_{3} \mathrm{OS}$ (267.31) : C,62.92; H,3.40. Found: C,62.93; $\mathrm{H}, 3.38 \%$.

General method for compounds of $5 a, b$

A mixture of 3a,b $(0.01 \mathrm{~mol})$ and ethyl cyanoacetate $(0.015 \mathrm{~mol})$ was heated at $130-140{ }^{\circ} \mathrm{C}$ in dimethylformamide $(10 \mathrm{ml})$ for $4 \mathrm{hr}$, the reaction mixture was then 1 cooled to room temperature and ether $(30 \mathrm{ml})$ was added. the solid precipitate was collected and washed several times with water and recrystallized from a suitable solvent to give 5a,b.

2-(6-(benzo[d]thiazol-2-yl)-2,3-dihydrobenzo[d]thiazol-2-yl) acetonitrile(5a)

Yellow solid (71\% yield) m.p. $147-150^{\circ} \mathrm{C}$ IR $(\mathrm{KBr}) \mathrm{cm}^{-1} ; 3067$ (aromatic$\mathrm{CH}$ ), 2368(CN), 1600, 1455(CH-aromatic); ${ }^{1} \mathrm{H}$ NMR (DMSO-d6) $\delta: 4.32$ (d, 2H, $\left.\mathrm{CH}_{2}\right), 6.39-8.23(\mathrm{~m}, 7 \mathrm{H}, \mathrm{ArH})$. Anal. Calculated for $\mathrm{C}_{16} \mathrm{H}_{9} \mathrm{~N}_{3} \mathrm{~S}_{2}(307.39)$ :C,62.56, H,2.94. Found: C, 62.56; H,2.94.\%

2-(6-(benzo[d]oxazol-2-yl)benzo[d]thiazol-2-yl)acetonitrile(5b)

Yellow solid, (50\% yield) m.p.290-292 ${ }^{\circ}$; IR $(\mathrm{KBr}) \mathrm{cm}^{-1}: 3053$ (aromatic$\mathrm{CH}), 2368(\mathrm{CN}), 1600,1455$ (aromatic ring system); ${ }^{1} \mathrm{H}$ NMR; $\delta: 4.00(\mathrm{~s}, 2 \mathrm{H}$, $\mathrm{CH}_{2}$ ), 7.5-7.7 (m, 7H, ArH), Anal. Calculated For $\mathrm{C}_{16} \mathrm{H}_{9} \mathrm{~N}_{3} \mathrm{OS}(291.33)$ :C,65.96 ;H,3.11 .Found:C,65.98; H,3.09\%.

General method for synthesis of compounds $6 a, b$

To a cold solution 1a,b $(0.03 \mathrm{~mol})$ in concentrated hydrochloric acid $(7.98 \mathrm{ml})$, diluted with $30 \mathrm{ml}$ water with stirring, was added a solution of sodium nitrite $(0.02 \mathrm{~mol})$ dissolved in $(80 \mathrm{ml})$ of water maintaining the temperature below $5^{\circ} \mathrm{C}$.The mixture was stirred for $30 \mathrm{~min}$ then added portion wise to a solution of copper (I) cyanide $(0.26 \mathrm{~mol})$ with stirring at 60 $70^{\circ} \mathrm{C}$ for $15 \mathrm{~min}$, then poured into water. The precipitate that formed was filtered and washed with water and re-crystallized from ethanol to give 6a,b.

\section{4-(benzo[d]thiazol-2-yl)benzonitrile(6a)}

Orang solid, (50\% yield), m.p. $119-220^{\circ} \mathrm{C}$; IR $(\mathrm{KBr}) \mathrm{cm}^{-1}$; 3053(aromatic$\mathrm{CH}), 2230(\mathrm{CN}) ;{ }^{1} \mathrm{H}$ NMR ; $\delta=7.57-8.23 \mathrm{ppm}(\mathrm{m}, 8 \mathrm{H}, \mathrm{ArH})$; Anal. Calculated For $\mathrm{C}_{14} \mathrm{H}_{8} \mathrm{~N}_{2} \mathrm{~S}$ (236.29): C, 71.16; H, 3.41. Found: $\mathrm{C}, 71.45 ; \mathrm{H}, 3.11 \%$.

\section{4-(benzo[d]oxazol-2-yl)benzonitrile(6b)}

Yellow solid (60\% yield), m.p. 223-225 ${ }^{\circ} \mathrm{C}$; IR (KBr) cm ${ }^{-1}, 3029$ (aromatic$\mathrm{CH}), 2250(\mathrm{CN})$; $1 \mathrm{H}$ NMR $\delta: 7.75-8.23 \mathrm{ppm}(\mathrm{m}, 8 \mathrm{H}, \mathrm{ArH}) ; . \mathrm{MS} ; \mathrm{m} / \mathrm{z}(\%) 221$ $\left(\mathrm{M}^{+}, 100 \%\right)$, , (192 ,15\%), (118,5\%), (92,11\%), (64,53\%). Anal. Calculated For $\mathrm{C}_{14} \mathrm{H}_{8} \mathrm{~N}_{2} \mathrm{O}$ (220.06): C, 73.50; H, 4.10, Found: C, 73.81; H, $4.61 \%$.

\section{General method for synthesis of compounds $7 a$ and $b$}

A mixture of $6 \mathrm{a}, \mathrm{c}(0.01 \mathrm{~mol})$ and thioglycolic acid $(0.01 \mathrm{~mol})$ in $20 \mathrm{ml}$ glacial acetic acid was refluxed for $5 \mathrm{hr}$, allowed the reaction mixture to cool then 
poured into water. The precipitate was collected and washed several times with water and re-crystallized from ethanol to give $7 \mathrm{a}, \mathrm{c}$.

2-(4-(benzo[d]thiazol-2-yl) phenyl) thiazol-4(5H)-one (7a)

Orang solid, (65\% yield), m.p $128-130^{\circ} \mathrm{C}$; IR $(\mathrm{KBr}) \mathrm{cm}^{-1}: 3045(\mathrm{CH}-$ aromatic), $1645(\mathrm{C}=\mathrm{O}) ;{ }^{1} \mathrm{HNMR}$ (DMSO-d $\left.d_{6}\right) \delta: 3.81\left(\mathrm{~s}, 2 \mathrm{H}, \mathrm{CH}_{2}\right), 7.6-8.4(\mathrm{~m}$, $8 \mathrm{H}$, aromatic protons). Anal. Calculated For $\mathrm{C}_{16} \mathrm{H}_{10} \mathrm{~N}_{2} \mathrm{OS}_{2}$ : (310.02), C,61.91; H, 3.25. Found: C,62.14; H,2.90\% .

2-(4-(benzo[d]oxazol-2-yl) phenyl) thiazol-4(5H)-one (7b)

Yellow solid, (60\% yield), m.p 278-280 ${ }^{\circ}$; IR (KBr) cm $\mathrm{cm}^{-1}: 3225(\mathrm{NH}), 3045$ $\left(\mathrm{CH}\right.$ aromatic), 1645(C=O); ${ }^{1} \mathrm{H}$ NMR(DMSO-d6) $\delta=3.98(\mathrm{~s}, 2 \mathrm{H}, \mathrm{CH} 2), 7.60$ $8.4 \mathrm{ppm} \quad(\mathrm{m}, \quad 8 \mathrm{H}$, aromatic protons Anal. Calculated for $\mathrm{C}_{16} \mathrm{H}_{10} \mathrm{~N}_{2} \mathrm{O}_{2} \mathrm{~S}(294.33): \mathrm{C}, 65.29 ; \mathrm{H}, 3.42$.Found:C,65.3;H,3.44\%.

General method for synthesis of componds of $8 a$ and $b$

A mixture of 4-(1,3-benzoazol-2-yl) benzonitrile 6a,b (0.006 mol) sodium azide $(0.007 \mathrm{~mol})$ and ammonium chloride $(0.007 \mathrm{~mol})$ dissolved in dimethylformamide, was refluxed in an oil bath at $120-130^{\circ} \mathrm{C}$ for about $24 \mathrm{hr}$, then cooled, poured into water and the precipitate was collected and recrystallized from dimethylformamide to give 8a,b.

2-(4-(1H-tetrazol-5-yl) phenyl)benzo[d]thiazole $(8 a)$

Orang solid, $\left(60 \%\right.$ yield), m.p. $293-295^{\circ} \mathrm{C}$; IR $(\mathrm{KBr}) \mathrm{cm}^{-1}$; 3193, 3064(NH),3049(aromatic-CH),1332-1115(C-N), 1108, 1138 (C=H- aromatic); ${ }^{1} \mathrm{HNMR} ; \delta=7.54-8.21(\mathrm{~m}, 8 \mathrm{H}, \mathrm{Ar}-\mathrm{H}), 8.52(\mathrm{~s}, 1 \mathrm{H}, \mathrm{NH})$. Anal. Calculated For $\mathrm{C}_{14} \mathrm{H}_{9} \mathrm{~N}_{5} \mathrm{~S}$ (279.32): C, 60.20; H, 3.25. Found: C, 60.50; H, 3.02\%.

2-(4-(1H-tetrazol-5-yl)phenyl)benzo[d]oxazole (8b)

Orang solid, $\left(65 \%\right.$ yield), m.p. $297-299^{\circ} \mathrm{C}$; IR $(\mathrm{KBr}) \mathrm{cm}^{-1}: 3193(\mathrm{NH})$, 3067(aromatic-CH), 13423-1032(C-N)1108,1138(C=H-Aromatic); $\quad \mathrm{H}^{1} \mathrm{NMR}$; $\delta=7.54-8.12(\mathrm{~m}, 8 \mathrm{H}, \mathrm{Ar}-\mathrm{H}), 4.3(\mathrm{~s}, 1 \mathrm{H}, \mathrm{NH}) ; \mathrm{MS} \mathrm{m} / \mathrm{z}(\%)$ Anal. Calcd. for $\mathrm{C}_{14} \mathrm{H}_{9} \mathrm{~N}_{5} \mathrm{O}$ (263.08): C, 63.87; H, 3.45. Found: C, 64.18; H, 3.14\%.

General method for synthesis of $9 a$ and $b$

A solution of 2-(4-(1H-tetrazol-5-yl)phenyl)benzo[d]X hydrochloride $8 \mathrm{a}, \mathrm{b}$ $(0.01 \mathrm{~mol})$ and acetic anhydride $(0.5 \mathrm{~mol})$ was refluxed at $150^{\circ} \mathrm{in}$ an oil bath for $3 \mathrm{hr}$, then cooled and poured into water the precipitate was collected and recrystallized from ethanol to give 9 a,b.

2-(4-(5-methyl-1, 3, 4-oxadiazol-2-yl) phenyl) benzo[d]thiazole (9a)

Orang solid, (75\% yield), m.p. $135-136{ }^{\circ} \mathrm{C}$; IR ( KBr) $\mathrm{cm}^{-1}, 3053$ (aromatic$\mathrm{C}-\mathrm{H}){ }^{1} \mathrm{HNMR} ; \delta=3.5\left(\mathrm{~s}, 3 \mathrm{H}, \mathrm{CH}_{3}\right), 7.3-8.5(\mathrm{~m}, 8 \mathrm{H}, \mathrm{ArH}) . \mathrm{MS} \mathrm{m} / \mathrm{z}(\%)$, $\mathrm{M}^{+}(293,100 \%), \quad \mathrm{M}^{+1}(294,10.8 \%), \quad \mathrm{M}^{+2}(295,1.4 \%)$, Anal. Calculated For $\mathrm{C}_{16} \mathrm{H}_{11} \mathrm{~N}_{3} \mathrm{OS}$ (293.34) : C, 65.51; H,3.78. Found: C, 65.53; H, 3.88\%. 
2-(4-(5-methyl-1, 3, 4-oxadiazol-2-yl) phenyl) benzo[d] oxazole (9b)

Orang solid, (75\% yield), m.p 294-295 ${ }^{\circ} \mathrm{C}$; IR ( KBr) cm${ }^{-1}, 3067$ (aromatic$\mathrm{CH}) ;{ }^{1} \mathrm{HNMR} ; \quad \delta=2.9\left(\mathrm{~s}, \quad 3 \mathrm{H}, \quad \mathrm{CH}_{3}\right), \quad 7.3-8.5 \quad(\mathrm{~m}, \quad 8 \mathrm{H}, \quad \mathrm{ArH}) . \quad \mathrm{MS} \quad \mathrm{m} / \mathrm{z}$ (\%), $\mathrm{M}(277,100 \%), \mathrm{M}^{+1}(278,1.9 \%)$, Anal.Calculated For $\mathrm{C}_{16} \mathrm{H}_{11} \mathrm{~N}_{3} \mathrm{O}_{2}(277.28)$ : C, 69.31; H, 4.00. Found: C, 69.32; H, $3.98 \%$.

General method for synthesis of $10 \mathrm{~b}$ and $c$

A solution of $(1 \mathrm{~b}, \mathrm{c})(0.2 \mathrm{~mol})$, triethylorthoformate $(0.2 \mathrm{~mol})$ and 4-methyl pyrazolone, $(0.2 \mathrm{~mol})$ in dimethylformamide in presence of triethylamine were heated under reflux in an oil bath at $140^{\circ} \mathrm{C}$ for about $2.5 \mathrm{hr}$. The alcohol was permitted to distill off from the reaction mixture and the solid residue was cooled and treated with petroleum ether $(40-60)^{0} \mathrm{C}$ to give $10 \mathrm{~b}, \mathrm{c}$.

4-((4-(benzo[d]oxazol-2-yl)phenylamino)methylene)-3-methyl-1H-pyrazol$5(4 H)$ - one $(10 b)$

White solid (75\% yield), m.p. $\quad 278-280{ }^{\circ} \mathrm{C}$; $\quad$ IR $(\mathrm{KBr}) \mathrm{cm}^{-1}, 3265$ $(\mathrm{NH}), 3050(\mathrm{Ar}-\mathrm{H}), 1648.6(\mathrm{CO}),{ }^{1} \mathrm{H}$ NMR; $\delta=3.4 \quad\left(\mathrm{~s}, 3 \mathrm{H}, \mathrm{CH}_{3}\right), 6.0(\mathrm{~s}, 1 \mathrm{H}$, $\mathrm{C}=\mathrm{H}), \quad 6.5(\mathrm{~s}, 1 \mathrm{H}, \mathrm{NH}-\mathrm{CH}), 6.52-7.26(\mathrm{~m}, 8 \mathrm{H}, \mathrm{ArH}) 8.5(\mathrm{~s}, 1 \mathrm{H}, \mathrm{NH}) \mathrm{D}_{2} \mathrm{O}$ exchangeable. MS m/z(\%)Anal. Calculated For $\mathrm{C}_{18} \mathrm{H}_{14} \mathrm{~N}_{4} \mathrm{O}_{2}$ (318.33): C, 67.91; H, 4.43; Found: C, 67.80; H, 4.40.\%

4-((4-(benzo[d]Imidazol-2-yl)phenylamino)methylene)-3-methyl-1H-pyrazol$5(4 H)$-one $(10 c)$.

White solid, (70\% yield), m.p. $258-260^{\circ} \mathrm{C}$; IR ( $\left.\mathrm{KBr}\right) \mathrm{cm}^{-1}: 3215(\mathrm{NH})$, 3040 (aromaticC-H), $1700(\mathrm{CO}),{ }^{1} \mathrm{H}$ NMR; $\delta=2.9\left(\mathrm{~s}, 3 \mathrm{H}, \mathrm{CH}_{3}\right), 5.86(\mathrm{~d}, 1 \mathrm{H}$, $=\mathrm{CH}) .6 .31(\mathrm{~d}, 1 \mathrm{H}, \mathrm{NH}) \mathrm{D}_{2} \mathrm{O}$ exchangeable, $6.7-7.8\left(\mathrm{~m}, 8 \mathrm{H}, \mathrm{ArH}+1 \mathrm{H}, \mathrm{NH}, \mathrm{D}_{2} \mathrm{O}\right.$ exchangeable), $8.1(\mathrm{~s}, 1 \mathrm{H}, \mathrm{NH}) \mathrm{D}_{2} \mathrm{O}$ exchangeable , Anal .Calculated For $\mathrm{C}_{18} \mathrm{H}_{15} \mathrm{~N}_{5} \mathrm{O}(317.34): \mathrm{C}, 68.13$; H, 4.76; Found: $\mathrm{C}, 68.14 ; \mathrm{H}, 4.72 . \%$.

General method for synthesis of 11a-d.

A solution of the appropriate compounds 1a-c $(0.2 \mathrm{~mol})$, triethyl orthoformate $(0.2 \mathrm{~mol})$ and active methylene compounds $(0.2 \mathrm{~mol})$ in dimethylformamide was heated under reflux for $2.5 \mathrm{hr}$, The alcohol was permitted to distil off from the reaction mixture. The solid formed was recrystallized from petroleum ether $\left(60-80^{\circ} \mathrm{C}\right)$ to give $11 \mathrm{a}-\mathrm{d}$.

2-((4-(benzo[d]thiazol-2-yl)phenylamino)methylene)malononitrile(11a)

Brown solid, (65 \% yield), m.p $296^{\circ}-299^{\circ} \mathrm{C}$; IR( $\left.\mathrm{KBr}\right) \mathrm{cm}^{-1}, 3215$ $(\mathrm{NH}), 3067$ (aromatic-CH), 2220(CN). ${ }^{1} \mathrm{H}$ NMR; $\delta=4.09(\mathrm{~s}, 1 \mathrm{H}, \mathrm{NH}), 6.8(\mathrm{~s}$, $1 \mathrm{H}, \mathrm{C}=\mathrm{H}), 7.30-7.70 .(\mathrm{m}, 8 \mathrm{H}, \mathrm{ArH})$.Anal. Calculated For $\mathrm{C}_{17} \mathrm{H}_{10} \mathrm{~N}_{4} \mathrm{~S}$ (302.53): C, 67.53; H, 3.33;N,18.53. Found: C, 67.45; H,3.1\% .

Ethyl 3-(4-(benzo[d]thiazol-2-yl)phenylamino)-2-cyanoacrylate(11b)

Black solid, (50\% yield), m.p 159-160 ${ }^{\circ} \mathrm{C}$; IR( $\left.\mathrm{KBr}\right) \mathrm{cm}^{-1} 3122(\mathrm{NH})$, $3090\left(\right.$ aromatic-CH), $2120(\mathrm{CN}), 1746(\mathrm{COO}), 1648.6(\mathrm{CO}) ;{ }^{1} \mathrm{H} \mathrm{NMR} ; \delta=2.36(\mathrm{t}$, $\left.3 \mathrm{H}, \mathrm{CH}_{3}\right), 3.80$ (q, 2H, $\mathrm{CH}_{2}$ ), 7.12 (s, $1 \mathrm{H}$ ethylene), 6.52-8.23 (m, 8H, ArH), 
$11.38(\mathrm{~s}, 1 \mathrm{H}, \mathrm{NH}) \mathrm{D}_{2} \mathrm{O}$ exchangeable. Anal. Calculated For $\mathrm{C}_{19} \mathrm{H}_{15} \mathrm{~N}_{3} \mathrm{O}_{2} \mathrm{~S}$ (349.41): C, 65,31; H, 4.33 Found: C, 65.29; H, 4.28\% .

2 - (4-(1H-benzo[d]imidazol-2-yl)phenylamino) methylene) malononitrile (11c)

Brown solid, (65\% yield), m.p. $210-212{ }^{\circ} \mathrm{C}$; IR ( $\left.\mathrm{KBr}\right) \mathrm{cm}^{-1}$ : 3058, $(\mathrm{NH}), 3174($ aromatic-CH), $), 2200(\mathrm{CN}), 1648.6(\mathrm{CO}) ;{ }^{1} \mathrm{HNMR} ; \delta=4.09(\mathrm{~s}, 1 \mathrm{H}$, $\mathrm{NH}), 5.8(\mathrm{~s}, 1 \mathrm{H}$, benzimidazole-H), $7.7(s, 1 \mathrm{H}, \mathrm{C}=\mathrm{H}), 7.30-7.70(\mathrm{~m}, 8 \mathrm{H}$, ArH); MS: m/z \% 285(M $\left.{ }^{+}, 100\right), 259$ (15.4), 234 (4.8), 208 (1.3), 181 (3.3), 117 (1.3), 90 (9.2), 63 (28.6). Anal .Calculated For $\mathrm{C}_{17} \mathrm{H}_{11} \mathrm{~N}_{5}$ (285.3): C, 71.57; H, 3.89; Found : C, 71.61; H, 4.00\%.

Ethyl-2-((4-(1H-benzo[d]imidazol-2-yl)phenylamino)methylene)-3oxobutanoate $(11 d)$

Orang solid, (75\% yield); m.p. 280-285 ${ }^{\circ} \mathrm{C}$; IR ( $\left.\mathrm{KBr}\right) \mathrm{cm}^{-1}: 3058$, $(\mathrm{NH}), 3000$ (aromatic-CH), $1648(\mathrm{CO}) ;{ }^{1} \mathrm{HNMR} ; \delta=1.31\left(\mathrm{t}, 3 \mathrm{H}, \mathrm{CH}_{3}\right), 2.0(\mathrm{~s}$, $\left.3 \mathrm{H}, \mathrm{CH}_{3}\right), 4.2\left(\mathrm{q}, 2 \mathrm{H}, \mathrm{CH}_{2}\right), 5.0(\mathrm{~s}, 1 \mathrm{H}, \mathrm{NH}) \mathrm{D}_{2} \mathrm{O}$ exchangeable, 6.49-7.21 (m, $4 \mathrm{H}, \mathrm{ArH}), 7.12(\mathrm{~s}, 1 \mathrm{H}, \mathrm{C}=\mathrm{H}), 7.26-7.70(\mathrm{~m}, 4 \mathrm{H}, \mathrm{ArH}), 10.7(\mathrm{~s}, 1 \mathrm{H}, \mathrm{NH}) \mathrm{D}_{2} \mathrm{O}$ exchangeable. Anal. Calculated for $\mathrm{C}_{20} \mathrm{H}_{19} \mathrm{~N}_{3} \mathrm{O}_{3}$ (349.38): C, 68.75; H, 5.48 Found: C, 68.81; H, 5.41\%

General method for synthesis of $12 a-e$

A solution of $1 \mathrm{a}-\mathrm{c}(0.2 \mathrm{~mol})$, triethylorthoformate $(0.2 \mathrm{~mol})$ and aromatic amine compounds $(0.2 \mathrm{~mol})$ in dimethylformamide were heated under reflux for about two and half hours the alcohol was permitted to distal off from the reaction mixture. The solution solidified and re-crystallized from petroleum ether $60-80{ }^{\circ} \mathrm{C}$ to give $12 \mathrm{a}-\mathrm{e}$.

$N$-(4-(benzo[d] thiazol-2-yl)phenyl)- $N^{\prime}$-(4-chlorophenyl)formamidine 12 a.

Black solid, $(60 \%$ yield $), \quad$ m.p.96- $99^{\circ} \mathrm{C} ; \operatorname{IR}(\mathrm{KBr}) \mathrm{cm}^{-1}$; 3194(NH),3050(aromatic-CH) 1562(C=N),835(C-CL); ${ }^{1} \mathrm{HNMR} ; \delta=4.8(\mathrm{~s}$, 1H, NH), 6.4-7.2 (m, 8H, ArH), 7.5(s,1H,methylene), 7.7-8.3 (m, 4H, ArH), . Anal. Calculated for $\mathrm{C}_{20} \mathrm{H}_{14} \mathrm{ClN}_{3} \mathrm{~S}(363.86)$ : C, 66.02; H, 3.88 . Found: C, $66.06 ; \mathrm{H}, \quad 4.02 \% \quad$ (-N-(4-(benzo[d]oxazol-2-yl)phenyl)-N'-(4chlorophenyl)formamidine $12 \mathrm{~b}$. Orang Solid, (65\% yield), m.p.220- $223{ }^{\circ} \mathrm{C}$; IR ( $\mathrm{KBr}) \mathrm{cm}^{-1}, \quad 3057(\mathrm{NH}), 3050($ aromatic-CH), $1602(\mathrm{C}=\mathrm{N}), 830(\mathrm{C}-\mathrm{CL})$; ${ }^{1} \mathrm{HNMR} ; \delta=4.3$ (s, 1H, NH), 6.5-7.2 (m,8H, ArH's), 7.3-7.4 (m, 4H, ArH) 7.80 (s, 1H, CH).Anal. Calculated for $\mathrm{C}_{20} \mathrm{H}_{14} \mathrm{ClN}_{3} \mathrm{O}(347.8)$ : C, 69.07; H, 4.06. Found: C, 69.05; H, $4.03 \%$.

$N$-(4-(1H-benzo[d]imidazol-2-yl)phenyl)-N'-(4-chlorophenyl)formamidine $12 c$

Orang solid, (70 \% yield), m.p. 110-115 ${ }^{\circ} \mathrm{C}$; IR (KBr) cm ${ }^{-1}, 3194,3057$ $(\mathrm{NH}), 1602(\mathrm{CN}), 789(\mathrm{C}-\mathrm{Cl})$; ${ }^{1} \mathrm{HNMR} \delta=5.59(\mathrm{~s}, 1 \mathrm{H}$, imidazole-H), 6.647.5(q, $4 \mathrm{H}$, phenyl protons $), 7.50(\mathrm{~s}, 1 \mathrm{H}, \mathrm{CH}=\mathrm{N}), 7.75-8.1(\mathrm{~m}, 8 \mathrm{H}, \mathrm{ArH})$ and 12.83 
(d, $1 \mathrm{H}, \mathrm{NH}) \mathrm{D}_{2} \mathrm{O}$. Anal. Calculated for $\mathrm{C}_{20} \mathrm{H}_{15} \mathrm{ClN}_{4}(346.81): \mathrm{C}, 69.26 ; \mathrm{H}$, 4.32. Found: $\mathrm{C}, 69.27 ; \mathrm{H}, 4.31 \%$.

\section{$N$-(4-(1H-benzo[d] imidazol-2-yl)phenyl)-N'-m-tolylformamidine $12 d$.}

Red solid, (50 \% yield), m.p.103-105 ${ }^{\circ} \mathrm{C}$; IR: 3194, $3057(\mathrm{NH}), 1602(\mathrm{C}=\mathrm{N})$, ${ }^{1} \mathrm{HNMR} ; \delta=3.4\left(\mathrm{~s}, 3 \mathrm{H}, \mathrm{CH}_{3}\right), 5.59(\mathrm{~s}, 1 \mathrm{H}, \mathrm{NH}), 6.64-7.5(\mathrm{q}, 4 \mathrm{H}, \mathrm{ArH}$ 's), 7.75-8.1 (m, 8H, Ar-H's), $10.37(\mathrm{~d}, 1 \mathrm{H}, \mathrm{CH}), 12.83(\mathrm{~d}, 1 \mathrm{H}, \mathrm{NH}) \mathrm{D}_{2} \mathrm{O}$ exchangeable. Anal. Calculated for $\mathrm{C}_{21} \mathrm{H}_{18} \mathrm{~N}_{4}(326.39)$ :C, 77.30; H, 5.52. Found: C, 77.29; H, 5.51.\%

$N$-(4-(benzo[d]thiazol-2-yl)phenyl)-N'-m-tolylformamidine12e

Yellow solid, (50 \% yield), m.p.200-210 ${ }^{\circ} \mathrm{C}$; IR: 3194, $3057(\mathrm{NH}), 1602$ $(\mathrm{C}=\mathrm{N}),{ }^{1} \mathrm{HNMR}, \delta=3.2\left(\mathrm{~s}, 3 \mathrm{H}, \mathrm{CH}_{3}\right), 6.68(\mathrm{~d}, 1 \mathrm{H}, \mathrm{CH}), 7.5-7.7(\mathrm{q}, 4 \mathrm{H}, \mathrm{ArH})$, 8.01-8.25 (m, 8H, ArH), $8.22(\mathrm{~d}, 1 \mathrm{H}, \mathrm{NH}) \mathrm{D}_{2} \mathrm{O}$ exchangeable. Anal. Calculated for $\mathrm{C}_{21} \mathrm{H}_{17} \mathrm{~N}_{3} \mathrm{~S}(343.44)$ : $\mathrm{C}, 73.44 ; \mathrm{H}, 4.96$. Found: C, 73.46; H, 4.95.\%

\section{Conclusion}

The synthesis of new 2-(4aminophenyl) benzothiazole/ benzoxazole/ benzimidazole heterocyclic ring systems was accomplished through simple routes and their antitumor activity have been investigated and reported,in this study.

Pharmacological evaluation of compounds 2-12 against nine cell lines revealed that these compounds possess high or moderate anti-tumor activities .

From the above antitumor screening results some structure activity relationships can be suggested .The presence of pyrazole moiety as in compound 10c named -4-((4-(benzo[d]Imidazol-2-yl)phenylamino)methylene)3-methyl-1H-pyrazol-5(4H)-one enhance the cytotoxic activity.

Also, the introduction of thiocyanate in benzenamine moiety as in compound $2 b$ named 4-(benzo[d]oxazol-2-yl)-2-thiocyanatobenzenamine exhibited strong antitumor activity against various cancer diseases.

\section{References}

1. Shirini, F., Mamaghani, M. and Seddighi, M., Catalyst Res. Chem. Intermed. 41, 5611-5619 DOI 10.1007/s11164-014-1685-7 (2015).

2. Mortimer,C.G., Wells, G. and Crochard J.P., et al. J. Med. Chem. 49,17985(2006).

3. Khan, M., Imran, S. and Iqbal Choudhary, M., European Journal of Medicinal Chemistry, 92, 387-400(2015).

4. Fazal, R., Samreen, Muhammad, T., Syed, M.S., Shahnaz, P., Momin, K., Alam, M.T., Mohammed, K. K. and Choudhary, I., Journal of the Chemical Society of Pakistan, 37 (1), 157-161(2015). 
5. Shi, D.F., Bradshaw,T.D. and Wrigley, S. et al., J. Med. Chem. 39, 3375-84(1996)

6. Hu ,W.P. Chen, Y. K. and Liao, C.C. et al., Bioorg. Med. Chem. 18, 6197207(2010).

7. Choi , S.J., Park, H.J and Lee, S.K et al., Bioorg. Med. Chem.14, 1229-35(2006).

8. Novak, M. and Chakraborty, M., .J. Phys. Org. Chem. 24, 960-8(2011).

9. Tasler, S. Mu ller, O. and Wieber, T. et al., Bioorg. Med. Chem. Lett.19, 1349-56(2009).

10. Tzanopoulou , S., Pirmettis ,I.C. and Patsis, G. G. et al., J. Med. Chem. 49, 540810(2006).

11. Hutchinson , Bradshaw ,T.D. and Matthews, C.S. et al., Bioorg. Med. Chem. Lett. 13, 471-4(2003).

12. Bradshaw, T.D., Steven, M.F.G and Westwell, A.D., Current Medicinal Chemistry, 8, 203-210(2001).

13. Bradshaw, T.D., Wrigley, S. and Shi, D.F. et al., Brit. J. Cancer, 77,74552(1998).

14. O'Brie, S.E., Browne, H.L., Bradshaw, T.D. and Westwell, A.D., Org. Biomol. Chem. 1,493-497(2003).

15. Manal, M.K., Sameeha, M.A. Abelegwad, M.A. Abd el Bakky, M.S. and E.A.Fatma, Der Pharma Chemica,8(1), 117-123(2016).

16. Soni,N. Soni, N. and Gupta, P., Der Pharma Chemica, 8(4),77-82,(2016).

17. Yurttas, L. and Tay, F.D., J. Enzyme Inhib. Med. Chem. 30(3), 458-65(2015).

18. Vuong, Q., Van, Bednarikova, Z. and Antosova, A., J. Med. Chem. Commun. 6, $810(2015)$

19. Kova, J., Hamul'aková, S., Fedoročko, P., Kuča, K. and Kožurková, M., Eur. J. Pharm. Sci. 76, 192(2015).

20. L. Y.Funda Tay and S. Demirayak, Journal of Enzyme Inhibition and Medicinal Chemistry, DOI: 10.3109/14756366.2014.945168(2014).

21. Singh, M., Singh, S.K., Gangwar, M. G., Singh, S., Med. Chem. Res. 25, 263282(2016).

22. Bradshaw, T.D. and Westwell, A.D., Curr. Med. Chem, 11, 1241-53(2004).

23. Moharram, H.H., AbedFattah, A. and Manhey, F.M., Egypt. J. Chem. 26, 261263(1983)

24. Moharram, H.H., Arch. Pharm. Res. 13(1),14(1990). 
25. Hein, D.W., Alheim, R.J. and Leavitt, J.J., Am. Chem. Soc. 79(2), 427-429(1957).

26. Jursic, B.S. and Zdravkovskif, Z., Synthetic Communications, 24(11), (1994).

27. Boyd, M.R., Status of the NCI preclinical antitumor drug discovery screen. In: "Cancer: Principles and Practice of Oncology Updates" DeVita Jr, V.T., Hellman, S., Rosenberg, S.A. (Ed), pp.1-12, Vol. 3, No. 10. Philadelphia: JPLippincott (1989).

28. Weinstein, J.N., Myers, T.G., Oconnor, P.M., Friend, S.H., Fornace, A.J., Kohn, K.W., Fojo, T., Bates, S.E., Rubinstein, L.V., Anderson, N.L., Buolamwini, J.K., VanOsdol, W.W., Monks, A.P., Scudiero, D.A., Sausville, E.A. Zaharevitz, D.W. Bunow, B., Wittes, R.E and Paul, K.D. Science, 275,343(1997).

29. Marks, A., Scudiero, D. and Kehan, P.S. et al., Feasibility of high Flux AntiCancer Drug .Screen using a Diverse Panel of cultured Human Tumor cell lines. J. Nat. Caner Inst. 83,757(1991).

(Received 16/10/2016 ; accepted 29/11/2016) 
تثييبا وتقييم الفاعلية المضادة للاورام لمركبات جليدة من مشتقات ب (ع ـ امينوفينيل)

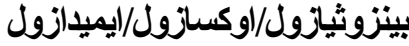

الهام محمد عز العرب' ، أحمد ابراهيم السيد ابراهيم' ، محاسن سعد أمين' و

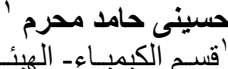

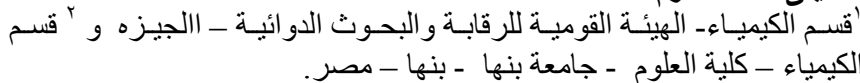

قد تم تثبيد مركبات متعددة من r -(ع ـأمينو فينيل) بينزوثيازول وكذلك مركبات

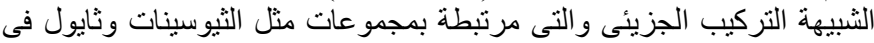

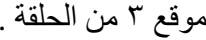

وقد تم تشييد مركبات مدموجة الحلقات البنزوثايزول ومركباتها المتشابهة

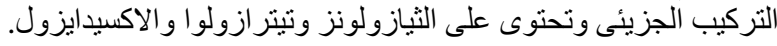

وقد تم تقييم و اختبار تلك المركبات الجديدة من حيث فاعليها ضد السرطان وقد أظهرت النتائج تاثير ات متفائلة ضد الخلايا السرطانية. 
